University of North Florida

UNIVERSITY of

NORTH FLORIDA.

UNF Digital Commons

\title{
Spin Dynamics In Single-Molecule Magnets Combining Surface Acoustic Waves And High-Frequency Electron Paramagnetic Resonance
}

F Macià

J Lawrence

S Hill

J M. Hernandez

J Tejada

See next page for additional authors

Follow this and additional works at: https://digitalcommons.unf.edu/achm_facpub

Part of the Chemistry Commons

\section{Recommended Citation}

Macià, F; Lawrence, J; Hill, S; Hernandez, J M.; Tejada, J; Santos, P V.; Lampropoulos, Christos; and Christou, G, "Spin Dynamics In Single-Molecule Magnets Combining Surface Acoustic Waves And HighFrequency Electron Paramagnetic Resonance" (2008). Chemistry Faculty Publications. 11.

https://digitalcommons.unf.edu/achm_facpub/11

This Article is brought to you for free and open access by the Department of Chemistry at UNF Digital Commons. It has been accepted for inclusion in Chemistry Faculty Publications by an authorized administrator of UNF Digital Commons. For more information, please contact Digital Projects.

(C) 2008 All Rights Reserved

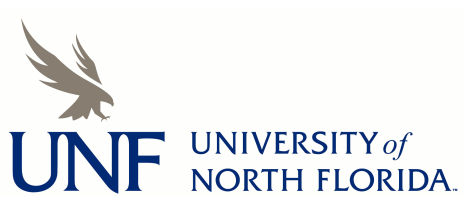




\section{Authors}

F Macià, J Lawrence, S Hill, J M. Hernandez, J Tejada, P V. Santos, Christos Lampropoulos, and G Christou 


\title{
Spin dynamics in single-molecule magnets combining surface acoustic waves and high-frequency electron paramagnetic resonance
}

\author{
F. Macià, ${ }^{1}$ J. Lawrence, ${ }^{2}$ S. Hill, ${ }^{2}$ J. M. Hernandez, ${ }^{1}$ J. Tejada, ${ }^{1}$ P. V. Santos,${ }^{3}$ C. Lampropoulos,${ }^{4}$ and G. Christou ${ }^{4}$ \\ ${ }^{1}$ Departament de Física Fonamental, Facultat de Física, Universitat de Barcelona, Avinguda Diagonal 647, Planta 4, \\ Edifici nou, 08028 Barcelona, Spain \\ ${ }^{2}$ Department of Physics, University of Florida, Gainesville, Florida 32611, USA \\ ${ }^{3}$ Paul-Drude-Institut für Festkörperelektronik, Hausvogteiplatz, 5-7, 10117 Berlin, Germany \\ ${ }^{4}$ Department of Chemistry, University of Florida, Gainesville, Florida 32611, USA
}

(Received 24 October 2007; revised manuscript received 30 November 2007; published 8 January 2008)

\begin{abstract}
We report an experimental technique that integrates high-frequency surface acoustic waves (SAWs) with high-frequency electron paramagnetic resonance (HFEPR) spectroscopy in order to measure spin dynamics on fast time scales in single-molecule magnets. After the system is driven out of equilibrium by triggering magnetic avalanches, or simply by heating with short SAW pulses, the evolution of the spin populations within fixed energy levels is measured using HFEPR spectroscopy.
\end{abstract}

DOI: 10.1103/PhysRevB.77.020403

PACS number(s): 75.50.Xx, 75.60.Jk, 76.30.-v

There is currently a large amount of research dedicated to understanding relaxation processes in single-molecule magnets. These materials have been proposed as possible candidates for quantum computers and high-density information storage devices. ${ }^{1,2}$ Before any technological applications can be implemented, however, one must have knowledge of the compound's interaction with the surrounding environment, and the mechanisms involved in establishing equilibrium in the system.

$\mathrm{Mn}_{12}$ acetate has been intensively studied after its magnetic bistability below $3.5 \mathrm{~K}$ was demonstrated. ${ }^{3}$ At low temperatures, a crystal of $\mathrm{Mn}_{12}$ acetate exhibits two modes of magnetic relaxation. The first manifests itself as a staircase hysteresis curve, which is due to thermally assisted quantum tunneling of the magnetization. ${ }^{4}$ The second relaxation mode is a much more rapid magnetization reversal that typically lasts a few milliseconds and only appears in sufficiently large crystals. It was first studied by Paulsen and Park ${ }^{5}$ and attributed to a thermal runaway or avalanche. ${ }^{6}$ In zero field, the ground $S=10$ state of $\mathrm{Mn}_{12} \mathrm{Ac}$ can be viewed as a doublewell potential, with an energy barrier separating states having equal magnitude but opposite spin projection onto the quantization $z$ axis. Figure 1 depicts the tilted potential barrier and illustrates with arrows the evolution of spins during an avalanche. Recent studies analyzed the stochasticity of the process, and the spatial dependence, treating the avalanches as a deflagration process. ${ }^{7}$ Additionally, a technique was proposed using surface acoustic waves (SAWs) to ignite and control the magnetic deflagration associated with the spin avalanches at a particular value of the applied magnetic field. ${ }^{8}$ Magnetic deflagration has been measured through magnetization with Hall bars, coils, and superconducting quantum interference devices (SQUIDs) ${ }^{7-9}$. Although these methods may involve spatial resolution, none of them allow analysis of the avalanche in a single energy level. The method we propose and discuss in this paper is based upon the use of microwave radiation to measure the spin dynamics in $\mathrm{Mn}_{12} \mathrm{Ac}$. High-frequency electron paramagnetic resonance (HFEPR) measurements probe the spin population differences between energy levels and hence our data provide energy-resolved information concerning how spins relax to equilibrium when an avalanche is triggered with a SAW pulse. In this Rapid Communication we discuss two different kinds of experiment where we have measured the thermal population changes between energy levels. The first involves magnetic avalanches and the second involves the use of small SAW pulses to perturb the equilibrium.

To produce the SAWs, we employed a special transducer design ${ }^{10}$ which yields devices capable of generating multiple harmonics of a fundamental frequency of $112 \mathrm{MHz}$, up to a maximum frequency of approximately $1 \mathrm{GHz}$. All experiments were performed using the third harmonic at $336 \mathrm{MHz}$, which was determined to be the optimum frequency to transmit the maximum power to the interdigital transducers (IDTs). A single crystal of $\mathrm{Mn}_{12}$ acetate, with dimensions approximately $0.3 \times 0.5 \times 0.5 \mathrm{~mm}^{3}$ was placed directly on the IDTs. This piezoelectric device was mounted on a copper block with a Cernox thermometer fixed approximately $10 \mathrm{~mm}$ from the device itself in order to monitor the temperature of the crystal. The copper block was attached to the end of a fundamental hybrid mode $\left(\mathrm{HE}_{11}\right)$ mode corrugated waveguide tube capable of propagating microwave radiation with almost no losses in the frequency range from

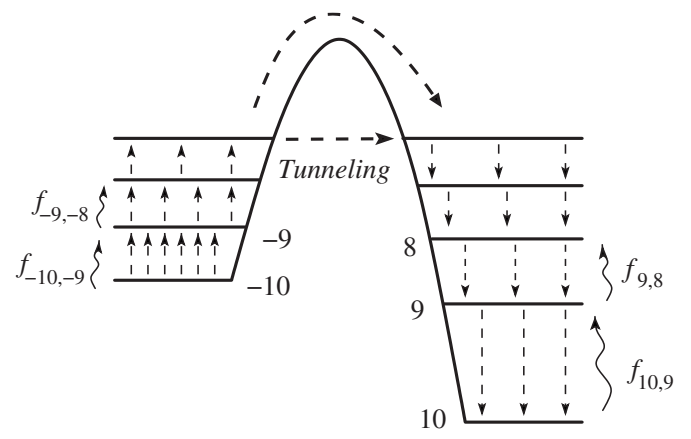

FIG. 1. Double-well potential diagram illustrating how spins relax during an avalanche. Spins in the right well relax to the ground state by emitting phonons, which result in heating of the system. This heating feeds back into the avalanche by exciting spins in the left well. 
180 to $330 \mathrm{GHz}$, with a maximum signal at $250 \mathrm{GHz}{ }^{11}$ The $5 \mathrm{~mm}$ opening where radiation exits the tube was positioned as close to the sample as possible ( $0.10 \mathrm{~mm}$ away) and centered over the sample to maximize coupling of the microwave power to the crystal. The corrugated tube was placed within the bore of a commercial superconducting magnet system capable of producing horizontal fields up to $7 \mathrm{~T}$ and temperatures in the range from 300 to $2 \mathrm{~K}$.

As a source and detector, we used a millimeter vector network analyzer (MVNA) associated with a Gunn source (tunable from 82 to $103 \mathrm{GHz}$ ) which was multiplied by a Schottky device giving powers of $1 \mathrm{~mW}$ at the third and fourth harmonics. A quasioptical bridge employing corrugated horns, a wire-grid polarizing beam splitter, and focusing mirrors was used to efficiently couple the spectrometer to the $\mathrm{HE}_{11}$ mode tube. The low microwave power represents an advantage of our technique since this does not cause measurable heating of the spins. Hence, the microwaves probe the system without disturbing the equilibrium. High sensitivity is achieved by phase-locking the Gunn source to the internal oscillators associated with the MVNA. In this way, the signal returned from the corrugated tube can be mixed down to $9 \mathrm{MHz}$ using a second Schottky device. This $9 \mathrm{MHz}$ signal was conditioned using a rf lock-in amplifier (SRS 844) with a time constant in the range $30-100 \mathrm{~s}$, and then recorded using a fast digitizer. In order to have the IDTs produce SAWs, we had to pulse the device at the corresponding megahertz frequency. Pulses from 1 s to $100 \mathrm{~ms}$ were used. We used a pulse pattern generator to trigger the SAW pulse and the fast data acquisition card simultaneously.

In our experiment, the sample is aligned with its magnetic easy axis approximately parallel $\left( \pm 1^{\circ}\right)$ to the applied field. With the temperature stabilized at $2.1 \mathrm{~K}$, we first apply a large magnetic field ( $3 \mathrm{~T}$ ) in order to magnetically polarize the sample, i.e., so that essentially all spins occupy the ground state $\left(m_{s}= \pm 10\right)$ of one of the two wells. The field is then reversed and held constant in the range between 0.45 and $1.1 \mathrm{~T}$, thus generating a metastable magnetization which can reverse either via tunneling or a thermal avalanche. Since the spin Hamiltonian parameters for $\mathrm{Mn}_{12}$ acetate are well known, ${ }^{12-15}$ we can tune the microwave radiation frequency to essentially any EPR transition within either the stable or metastable well (wavy arrows in Fig. 1) for a given magnetic field value; in practice, the bandwidth of the corrugated tube limits us to the lowest-lying transitions within each well. We then simultaneously trigger an avalanche (via a small SAW pulse) and the data acquisition card which records the EPR response of the sample via changes in the microwave signal reflected from the corrugated tube. The measurement is single shot in the sense that the avalanche is irreversible, i.e., no signal averaging is possible beyond that performed by the lock-in amplifier.

It is worth noting that there is a significant amount of heat released during an avalanche, and increases in temperature of between 6 and $12 \mathrm{~K}$ have been measured ${ }^{7,8}$ depending on the value of the applied magnetic field at which the avalanche occurs. This heating drives the system far out of equilibrium. Once all spins have avalanched, an elapsed time about a hundred milliseconds is needed to recover the initial bath temperature during which the excited spins slowly relax to

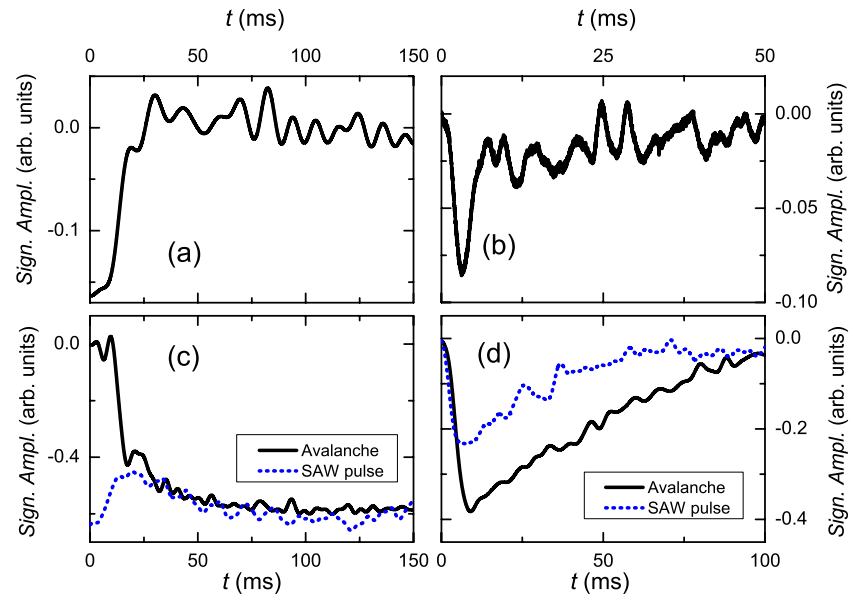

FIG. 2. (Color online) Evolution of the reflected microwave amplitude during an avalanche triggered via a SAW pulse. The EPR signals are presented in arbitrary units with a reference level of zero microwave absorption. In (a), $f=270 \mathrm{GHz}, \quad H=1.1 \mathrm{~T}$, and $m_{s}=10$ to 9 ; in (b), $f=237 \mathrm{GHz}, H=0.58 \mathrm{~T}$, and $m_{s}=9$ to 8 ; in (c) $f=320 \mathrm{GHz}, H=0.54 \mathrm{~T}$, and $m_{s}=10$ to 9 ; and in (d), $f=268 \mathrm{GHz}, H=0.54 \mathrm{~T}$, and $m_{s}=9$ to 8 . In (c) and (d), the dotted traces correspond to microwave absorption during a SAW pulse after the occurrence of the avalanche.

the ground state of the stable well. This time must be related with the phonon bottleneck effect; the phonons emitted by the direct process are absorbed again by the spins, resulting in a slower relaxation rate. It is widely believed that the bottleneck, a limiting spin-phonon relaxation effect, in this case is associated with the thermalization of the lattice, and is not characteristic of the intrinsic spin-lattice coupling. In our experiment, the main difference between EPR transitions observed in the metastable and stable wells is that the thermal bottleneck resulting from the heat release during the avalanche hardly affects the populations in the metastable well. These spins certainly populate higher-lying states, but only on the very short time scales characteristic of the avalanche ignition and propagation, i.e., they transit rapidly and irreversibly to the stable well. In contrast, populations within the stable well are strongly influenced by the bottleneck. Consequently, the evolution of EPR intensity associated with the two wells occurs on significantly different time scales: for the metastable well, this time scale is intrinsically related to the spin dynamics associated with the avalanche; in contrast, for the stable well, the spin population emerges rapidly, but the thermal inertia associated with the lattice results in a much slower relaxation back to the original bath temperature.

In the following figures, we plot the amplitude of the microwave signal reflected from the corrugated probe referenced to a level of zero microwave absorption (with arbitrary units). The signal drops below this reference level when the sample absorbs. Thus, the deviation from zero represents a measure of the spin populations associated with the levels involved in the chosen EPR transition. Figure 2 shows the time evolution of the absorption due to transitions from $m_{s}=10$ to 9 Fig. 2(a) and $m_{s}=9$ to 8 Fig. 2(b) in the metastable well after igniting an avalanche. In Fig. 2(a), the 
spins are initially in the metastable state, $S=10$, at $t=0$ and, therefore, absorb microwaves. After about $20 \mathrm{~ms}$ all of these spins have avalanched to the stable well, and there is no more absorption (within the oscillations of the noise). In Fig. 2(b) we can see that, at the beginning of the pulse $(t=0)$, there is no absorption. However, a sharp dip appears a few milliseconds after ignition of the avalanche resulting from the thermal excitation of spins from the metastable state, $S=10$. Initial and final EPR absorption values are the same because there is essentially no population in the $m_{s}=9$ level both before and after the avalanche. As a control, after recording data coincident with an avalanche, we pulse the IDT again in order to heat the sample with a pure SAW. No absorption is observed under these circumstances, as expected, since the avalanche has taken place and all spins have transited to the stable well while the frequency and magnetic field are tuned to EPR transitions in the metastable well.

Figures 2(c) and 2(d) show the EPR signals associated with the transitions from $m_{s}=10$ to 9 Fig. 2(c) and $m_{s}=9$ to 8 Fig. 2(d) in the stable well. In Fig. 2(c) we see initially that there is no absorption. However, there is a rapid increase in absorption during the avalanche, followed by a more gradual increase after its completion after the signal drops to zero in Fig. 2(a) . Figure 2(d) shows similar behavior at short times, followed by a slow decay in the EPR signal after the avalanche. Again, the avalanche takes some time to ignite (on the order of a few milliseconds after the trigger pulse), as can be seen from the EPR signals in Figs. 2(c) and 2(d), i.e., there is a slight delay before the onset of absorption. As the spins avalanche, they decay through the $m_{s}=9$ state before relaxing to the ground state $\left(m_{s}=10\right)$. The sharp, short-time sides of the absorption signals are characteristic of the fast (intrinsic) relaxation of spins from the metastable to the stable well. However, slower relaxation continues for approximately $100 \mathrm{~ms}$ after the avalanche. This can be understood from the fact that the avalanche releases a significant amount of heat into the lattice, which then takes a much longer time to reestablish equilibrium with the surrounding bath. During this process, spins follow the lattice temperature so that the $m_{s}=9$ state remains thermally populated and continues to absorb. Eventually, all spins relax to the $m_{s}=10$ state, and only the $m_{s}=10$ to 9 transition shows absorption. For comparison, after triggering an avalanche, we again pulse the IDT in order to heat the sample. The dotted traces in the figures illustrate the effect of this heat pulse. It is clear that there is a distinct difference in the EPR signals when comparing their evolution after triggering an avalanche and after a pure heat pulse. Careful analysis indicates that the slope of the signal after an avalanche is much steeper than that of the signal after pure SAW heating. Additionally, the SAW directly couples to the sample and the signal begins to change immediately, while the change of the signal due to an avalanche is delayed by a few milliseconds.

Other studies have shown that the thermal relaxation in SMMs is impeded by a phonon bottleneck. ${ }^{16}$ Data collected for transitions within the stable well support this assertion, and the associated times required to establish equilibrium ( $100 \mathrm{~ms}$ ) agree well with other studies. ${ }^{17}$ However, for transitions within the metastable well, we are able to observe faster dynamics which are not influenced by the bottleneck. ${ }^{18}$

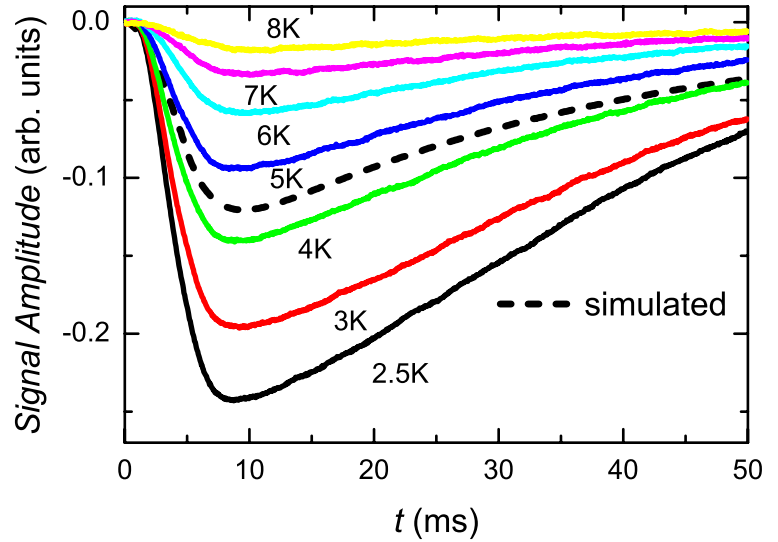

FIG. 3. (Color online) Time evolution of the EPR signal induced by SAW pulses at different temperatures from $T=2.5 \mathrm{~K}$ to $T=8 \mathrm{~K}$. The magnetic field is $H=6120 \mathrm{Oe}$, corresponding to a frequency difference, $f=269 \mathrm{GHz}$, between $m_{s}=9$ and $m_{s}=8$. The pulse duration is $5 \mathrm{~ms}$ and the beginning corresponds to $t=0$. The dashed line corresponds to a simulation of the data.

Consequently, the EPR signals resulting from these transitions probe spin population changes driven directly by the intrinsic coupling between the spins and the lattice during the propagation of the avalanche. Ultimately, our temporal resolution is limited by the velocity of the avalanche. Indeed, we can infer a spin response time on the order of $1 \mathrm{~ms}$, which is on the order of the time it takes for the avalanche to traverse the sample. This time, which agrees with other experiments in $\mathrm{Mn}_{12}$ acetate, ${ }^{7,8}$ nevertheless sets an upper limit for the spin-lattice relaxation time $T_{1}$.

A second experiment involved using short SAW pulses to heat the spins in the stable well (dotted traces in Fig. 2). This obviously does not trigger an avalanche but does weakly perturb the system such that higher-lying energy states become populated briefly before relaxing back to the ground state. We again monitor these changes in spin populations using low-power microwaves in order to probe specific EPR transitions within the stable well. The advantage of this technique is that it is repeatable, thereby permitting averaging of the data obtained for a train of heat pulses in order to improve the signal-to-noise ratio. Pulses of 1 s $50 \mathrm{~ms}$ and nominal power of $6 \mathrm{~dB} \mathrm{~m}$ were used, and experiments were repeated for a number of different bath temperatures.

Figure 3 shows a plot of the EPR signal as a function of time for the $m_{s}=9$ to 8 transition during and after application of a $5 \mathrm{~ms}$ heat pulse. In contrast to the signal trigged by an avalanche, the data here evolve smoothly without any sharp changes. As soon as the pulse is applied (at $t=0$ ) there is an increase in absorption due to thermal population of the $m_{s}=9$ state. This increase continues until the heat pulse is switched off, after which, the slower relaxation associated with the phonon bottleneck is observed. In order to quantify the observed behavior we assume that there are two important temperatures during the relaxation process: ${ }^{19}$ the lattice temperature $T_{l}$ and the spin temperature $T_{s}$, with respective relaxation time constants $l$ and $s \cdot l$ is related to lattice temperature variations when the heat pulse is switched on and off, and ${ }_{s}$ corresponds to the time spins take to follow 
these lattice temperature, which should ultimately be related to the spin lattice relaxation time, $T_{1}$. The dashed curve in Fig. 3 represents a simulation of the data using the equations described in Ref. 19. We deduce, $l$ decay times on the order of 10-50 ms (Ref. 19) and ${ }_{s}$ values which are 10-100 times faster than ${ }_{l}$. This suggests that ${ }_{s}$ is on the order of a few hundred microseconds, which agrees with the avalanche experiments and enables an estimate for an upper bound of a few hundred microseconds for $T_{1}$ in this system.

In conclusion, we have demonstrated a technique to monitor spin population dynamics by combining the use of SAWs and HFEPR. We are able to probe spin relaxation on reasonably fast times scales for specific spin quantum levels. By measuring the lifetimes of states within both the metastable and stable wells during the propagation of an avalanche, we can obtain information about the spin-lattice relaxation mechanisms in SMMs. Our results indicate an upper bound of a few hundreds of microseconds for the spin-lattice relaxation time $T_{1}$ in a single crystal of $\mathrm{Mn}_{12} \mathrm{Ac}$.

F.M. thanks the Spanish Ministerio de Educación y Ciencia for a research grant. S.H. acknowledges the support of the U.S. National Science Foundation (Grants No. DMR0239481 and No. DMR0414809). J.M.H. thanks the Ministerio de Educación y Ciencia and the Universitat de Barcelona for a Ramón y Cajal research contract.
${ }^{1}$ M. N. Leuenberger and D. Loss, Nature (London) 410, 789 (2001)

${ }^{2}$ J. Tejada, E. M. Chudnovsky, E. del Barco, J. M. Hernandez, and T. P. Spiller, Nanotechnology 12, 181 (2001).

${ }^{3}$ R. Sessoli, D. Gatteschi, A. Caneschi, and M. A. Novak, Nature (London) 365, 141 (1993).

${ }^{4}$ J. R. Friedman, M. P. Sarachik, J. Tejada, and R. Ziolo, Phys. Rev. Lett. 76, 3830 (1996).

${ }^{5}$ C. Paulsen and J.-G. Park, Quantum Tunneling of Magnetization, edited by L. Gunther and B. Barbara (Kluwer, Dordrecht, 1995), pp. 189-207.

${ }^{6}$ E. del Barco, J. M. Hernandez, M. Sales, J. Tejada, H. Rakoto, J. M. Broto, and E. M. Chudnovsky, Phys. Rev. B 60, 11898 (1999).

${ }^{7}$ Y. Suzuki et al., Phys. Rev. Lett. 95, 147201 (2005).

${ }^{8}$ A. Hernández-Mínguez, J. M. Hernandez, F. Macià, A. GarcíaSantiago, J. Tejada, and P. V. Santos, Phys. Rev. Lett. 95, 217205 (2005).

${ }^{9}$ C. H. Webster, O. Kazakova, J. C. Gallop, P. W. Josephs-Franks, A. Hernandez-Minguez, and A. Y. Tzalenchuk, Phys. Rev. B 76, 012403 (2007).

${ }^{10}$ K. Yamanouchi, C. H. S. Lee, K. Yamamoto, T. Meguro, and H.
Odagawa, Proceedings of the 1992 IEEE Ultrasonics Symposium (IEEE, New York, 1992), p. 139.

${ }^{11}$ R. S. Edwards, S. Hill, P. Goy, R. Wylde, and S. Takahashi, Physica B 346-347, 211 (2004).

${ }^{12}$ I. Mirebeau, M. Hennion, H. Casalta, H. Andres, H. U. Gudel, A. V. Irodova, and A. Caneschi, Phys. Rev. Lett. 83, 628 (1999).

${ }^{13}$ Y. C. Zhong, M. P. Sarachik, J. R. Friedman, R. A. Robinson, T. Kelley, H. Nakotte, A. Christianson, F. Trouw, S. M. J. Aubin, and D. N. Hendrickson, J. Appl. Phys. 85, 5636 (1999).

${ }^{14}$ S. Hill, R. S. Edwards, S. I. Jones, N. S. Dalal, and J. M. North, Phys. Rev. Lett. 90, 217204 (2003).

${ }^{15}$ E. del Barco, A. D. Kent, J. North, N. Dalal, E. Rumberger, D. Hendrickson, N. Chakov, and G. Christou, J. Low Temp. Phys. 140, 119 (2001).

${ }^{16}$ R. Schenker, M. N. Leuenberger, G. Chaboussant, D. Loss, and H. U. Güdel, Phys. Rev. B 72, 184403 (2005).

${ }^{17}$ K. Petukhov, S. Bahr, W. Wernsdorfer, A-L. Barra, and V. Mosser, Phys. Rev. B 75, 064408 (2007).

${ }^{18}$ A. Abragam and B. Bleaney, Electron Paramagnetic Resonance of Transition Ions (Dover, New York, 1970).

${ }^{19}$ J. M. Hernandez, F. Macià, A. Garcia-Santiago, J. Tejada, and P. V. Santos, Appl. Phys. Lett. 88, 012503 (2006). 